\title{
Whole Body Irradiation Induces Cutaneous Dendritic Cells Depletion via NF-kB Activation
}

\author{
Yanyong Yanga Jianguo Cui ${ }^{a} \quad$ Fu Gao Bailong Li Cong Liu Pei Zhang \\ Yijuan Huang Wen Liu Hu Liu Jianming Cai \\ Department of Radiation Medicine, Faculty of Naval Medicine, Second Military Medical University,

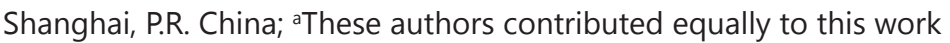

\section{Key Words}

Whole body irradiation $\cdot$ Dendritic cells $•$ Migration $・ N F-K B$

\begin{abstract}
Background: Effect of ionizing radiation on cutaneous dendritic cells (CDC) is critical to its influence on immune status of the skin, which plays an important role in the progression and recovery of radiation skin sickness. This study was to study the influence of whole body irradiation (WBI) on the CDC. Methods: Density of epidermal and dermal DC was determined with a fluorescent microscopy and the DC numbers in lymph node were measured by flow cytometry. A FITC induced migration assay was also used to study the migration of DC. The expressions of cytokines and chemokines were evaluated by Realtime PCR, and the protein level of was measured by Western blot. Results: WBI caused depletion of CDC in epidermal as well as dermal and augmented FITC-induced migration of DC to the draining lymph node $(\mathrm{LN})$. The number of DC migrated from ear explants to the CCL19-containing medium also increased after exposure to WBI. It was also found that WBI increased mRNA level of CCL19/ CCL21 as well as CCR7 in LN and skin tissue. The expressions of TNF $\alpha, I L-1 \alpha, I L-1 \beta$, and IL- 6 in skin tissues were also greatly induced by WBI in a dose dependent manner. Finally, we found that WBI induced translocation of nuclear factor $\mathrm{KB}(\mathrm{NF}-\mathrm{KB})$ and that the radiation-induced migration of DC was blocked by NF-KB inhibitor or TLR4 knockout. Conclusion: WBI caused $C D C$ depletion through induction of $D C$ migration to the draining $L N$, which might result from the activation of NF-KB and the induction of inflammatory microenvironment within the skin.
\end{abstract}




\section{Introduction}

Exposure to ionizing radiation (IR) can cause radiation skin injury, which could induce cellular alterations and inflammation in the epidermis and dermis. Even a year after exposure, the skin is still thin, hypovascularized and susceptible to trauma or infection. Besides an effective physical barrier, the skin also constitutes an immunological barrier against the surrounding environment [1]. The influence of IR on immune status is critical to its damages to the skin.

Interstitial dendritic cells in the dermal (dDC) and the Langerhans cells (LC) in the epidermal, known as cutaneous dendritic cells (cDC), are the most potent antigen presenting cells which could regulate immune response and induce self-tolerance in the skin. Both LC and $\mathrm{dDC}$ steadily migrates from the skin to the draining lymph node in a low rate [2]. And the migration is greatly enhanced by antigen or other inflammatory stimuli, which ensures an effective initiation of adaptive immune response [3]. Migration of DC is a highly regulated process which requires the participation of cytokines, chemokine, and some lipid mediators [4].

Cole et al. [5] reported that long-term local high dose of irradiation caused LC depletion from mouse footpads, and it was also demonstrated that LC in mouse ears was depleted after local irradiation [6]. Recently, Ryan et al. found that sub-lethal irradiation induced cDC migration from skin to the draining LN [2], while our department had found that ionizing radiation inhibited the migration capacity of DC in vitro [7]. Furthermore, as occupational exposure is becoming a major part of the radiation exposure, the migration capacity of cDC after moderate dose of whole body irradiation (WBI) is drawing more and more attention. In the present study, we demonstrated that different doses of WBI triggered cDC migration through a NF- $\kappa$ B mechanism.

\section{Materials and Methods}

\section{Mice and treatment}

All the experiments were approved by the Second Military Medical University, China in accordance with the Guide for Care and Use of Laboratory Animals published by the US NIH (publication No. 96-01). Female wild-type C57BL/6 mice, 4-6 weeks old, were purchased from China Academy of Science (Shanghai, China), Toll like receptor 4 knock out (TLR4-/-) mice were purchased from Model Animal Research Center of Nanjing University. Mice were housed in individual cages in a temperature-controlled room with a $12 \mathrm{~h}$ light/dark cycle. Food and water were provided ad libitum. Mice were irradiated in a holder designed to immobilize unanaesthetized mice to ensure the accuracy of radiation doses. For inhibition of the nuclear factor $\kappa \mathrm{B}(\mathrm{NF}-\kappa \mathrm{B})$ in vivo, mice were intra-peritoneally injected with ammonium pyrrolidinedithiocarbamate (PDTC) (Sigma, USA) at the concentrations of $10 \mathrm{mg} / \mathrm{kg}, 50 \mathrm{mg} / \mathrm{kg}$ and $100 \mathrm{mg} / \mathrm{kg} 1 \mathrm{~h}$ before irradiation. After different time post-irradiation, mice were killed by cervical dislocation and used in next experiments.

\section{Irradiation}

$60 \mathrm{Co}-\gamma$ rays in Irradiation Center (Faculty of Naval Medicine, Second Military Medical University, China) were used for the irradiation purpose. Mice were exposed to different doses of radiation, depending upon requirement of the present study.

\section{FITC-induced migration assay}

Immediately after $0,2,4,6$ Gy $\gamma$-irradiation, mice were painted with FITC on the area of the skin draining to inguinal LN as previously described [8]. Briefly, mice were anesthetized by using methoxyflurane and then the skin was clipped. FITC (Sigma, Saint Louis Missouri, USA) (8mg/ml) was dissolved in acetone and dibutyl phthalate (1:1) and applied with $25 \mathrm{ul}$ on each side of mice skin. $18 \mathrm{~h}$ later, the draining inguinal lymph node was isolated and digested with collagenase (Sigma) $(2 \mathrm{mg} / \mathrm{ml})$ for $20 \mathrm{~min}$ at $37^{\circ} \mathrm{C}$. Then cell suspension was prepared by passing through a fine wire mesh into a $35 \times 10 \mathrm{~mm}$ petri dish containing $1 \mathrm{ml}$ PBS. Isolated lymphocytes were washed 2 times with PBS. Then the cells were stained with PE-conjugated 
Yang et al.: Whole Body Irradiation and Dendritic Cells Migration

Table 1. PCR Primers

\begin{tabular}{lll}
\hline NAME & FORWARD & REVERSE \\
\hline CCL21 & aagaaccggggaacctctaa & tcctcttgagggctgtgtct \\
CCL19 & gtgcctgctgttgtgtgagt & cctgtagccagaggttggag \\
CCR7 & tttgttcacacaccccaga & tcagcgttcgaggttcttt \\
TNF $\alpha$ & catgcgtccagctgactaaa & tccccttcatcttcctcctt \\
IL-1 $\alpha$ & tcaaagcccaaaggaagcta & agctgactgctctggggata \\
IL-1 $\beta$ & ggcactttccagacttgctc & ctgttgagggattgggaaga \\
IFN $\gamma$ & acttgcttctgcctttcca & acaaggtcacccacaggaag \\
IL-6 & gctgacctctggacgcttac & gggagatgctttgttccaa \\
GAPDH & ggtgcctgtcgttgtgttc & gctccttctggtgctgttg \\
\hline
\end{tabular}

anti-CD11c mouse antibody (Ebioscience, Ireland, UK) and analyzed by flow cytometry (Beckman Coulter, California, USA).

Preparation of skin epidermal sheets and fluorescent microscopy

On different days after irradiation, the dorsal and ventral halves of removed mouse ears were split mechanically and the ventral halve was floated on $20 \mathrm{mM}$ EDTA/PBS at $37^{\circ} \mathrm{C}$ for $90 \mathrm{~min}$, and then epidermal sheets were removed [3]. The sheets were then fixed with $2 \%$ paraformaldehyde, rehydrated in PBS, blocked with PBS/1\% BSA, stained with PE-conjugated anti-CD11c mouse antibody (1:200) for 30min at room temperature and rehydrated in PBS again.

Cryosections (8um thick) were fixed in 2\% paraformaldehyde and stained with mouse anti-MHC $\alpha$ antibody (1:100) (Abcam, Cambridge, UK) followed by anti-mouse IgG secondary antibody (1:200) (Cell signaling, MA, USA) as previous described. Images were obtained using an Olympus BX60 fluorescent microscope equipped with a Retiga 2000R digital camera. Methods to evaluate DC density described in previous study were followed [9].

Flow cytometry analysis

On the 3rd day post-irradiation, auricular draining lymph node (ADLN) were isolated and digested with collagenase (Sigma) $(2 \mathrm{mg} / \mathrm{ml})$ for $20 \mathrm{~min}$ at $37^{\circ} \mathrm{C}$. After digestion, single cell suspensions were prepared by passing through a fine wire mesh into a $35 \times 10 \mathrm{~mm}$ petri dish containing $1 \mathrm{ml}$ PBS. Cells were then incubated with PE-conjugated mouse CD11c antibody and analyzed with flow cytometry (Beckman Coulter).

\section{Skin explants culture}

Methods previously described for evaluating DC migration using mouse skin explants were followed [10]. Immediately after irradiation, ears were removed and briefly washed in $70 \%$ ethanol and then placed ventral side down and split, removing the dorsal skin from the cartilage. Dorsal skin was placed split side down in $1 \mathrm{~mL}$ RPMI 1640 medium containing $10 \%$ fetal calf serum for $4 \mathrm{~h}$ at $37^{\circ} \mathrm{C}$ in a $5 \% \mathrm{CO}_{2}$ in air incubator to eliminate the non-DCs initially released. The skin was then transferred to another $1 \mathrm{~mL}$ RPMI 1640 medium, this time containing 0.1 ug recombinant mouse CCL19. After $24 \mathrm{~h}$-incibation at $37^{\circ} \mathrm{C}$, the cells that had migrated to medium were harvested and kept in $4^{\circ} \mathrm{C}$. The skin was transferred to fresh warm medium containing CCL19 and then incubated another 24h. Cells migrated from the skin over both incubations were pooled and counted by flow cytometry (Beckman Coulter, California, USA).

\section{Real time PCR}

Total RNA from skin and LN were extracted at different time points after irradiation using an RNA isolate kit (Takara, Dalian, China). cDNA was synthesized with a Reverse Transcript Kit (Takara, Dalian, China). And then realtime PCR were conducted on a quantified machine (Roche, Switzerland). All the experiments were administrated following the instructions of the kits. The primers we used in our experiments were shown in Table 1.

Western blot analysis

At $0,0.5,1,2 \mathrm{~h}$ post-irradiation, total protein and nuclear protein was extracted from skin tissues by using ProteoJETTM Mammalian Cell Lysis Reagent (Fermentas) and Nuclear Protein Isolation Kit (Beyotime, 
Haimen, China) respectively, according to the manufacture' protocols. Then Western blotting [11] was used to detect NF- $\mathrm{kB}$ p65 (Cell Signaling Technology, MA, USA; 1:1000) in each samples. $\beta$-actin (Cell Signaling Technology, MA, USA; 1:1000) was used as control for total protein, and lamin (Cell Signaling Technology, MA, USA, 1:1000) for nuclear protein. The secondary antibody (1:10000) was also purchased from CST.

Statistical analysis

Data are expressed as means \pm SEM for each experiment. The number of samples is indicated in the description of each experiment. We used an analysis of variance (ANOVA) followed by a Student-NewmanKeuls post hoc test for statistical analysis. We performed experiments for quantification in a blinded fashion.

\section{Results}

WBI induced epidermal and dermal DC depletion and migration

To study the influence of WBI on the density of DC in the epidermal, mice were irradiated and DC in the skin was labeled with CD11c+ antibody. The numbers of CD11c+ cells in epidermal decreased significantly at day 3 after 4Gy irradiation (Fig. 1A, B), and began to recover from day 5 (Fig. 1B). Our data also showed that CD11c+ cells per draining LN increased greatly at the 10th day after WBI (Fig. 1C). In the dermal, the density of MHC $\alpha+$ cells also declined after 2, 4, 6Gy irradiation (Fig. 1D). These data indicated that WBI induced DC migration from skin to the draining LN. By using a FITC-induced DC migration assay, we found that at $18 \mathrm{~h}$ after FITC painting, the number of CD11c+FITC+ cells in the draining LN of mice in the 2 Gy and 4Gy WBI group was significantly higher than the control group, while the 8Gy group showed no significant difference compared with the control group (Fig. 1E).

\section{WBI augmented DC migration from ear explants to CCL19}

A skin explant culture assay was used to determine whether WBI affects DC migration to the same concentration of CCL19 in the medium. After incubation in the CCL19-containing medium, cells migrated into the medium were counted. We found that 2Gy and 4Gy WBI significantly increased the number of cells in the medium compared with the shammed group, while 6Gy WBI group did not show a significant difference (Fig. 2), indicating that the migration capacity of DC and their responsiveness to CCL19 were enhanced by moderate doses of WBI.

WBI affected the mRNA level of CCR7 and CCL19/21

CCR7 and its ligands CCL19/21 are essential to DC's migration to LN from the skin. And expressions of CCR7 and CCL21 in skin after different doses of irradiation were measured. The mRNA level of CCR7 increased dramatically at $4 \mathrm{~h}$ after irradiation, while no difference was observed at $2 \mathrm{~h}$ or $8 \mathrm{~h}$ (Fig. 3A). WBI also hugely enhanced the level of CCL19 and CCL21 in skin tissues, but the level in LN changed only at 8h after irradiation (Fig. 3B, C). The induction of CCL19 and CCL21 by WBI was related to the radiation doses (Fig. 3D).

WBI caused up-regulation of inflammatory cytokines

Ionizing radiation induces inflammatory cytokines in skin tissues and many of them are involved in regulating the migration of DC. After 2, 4, 6Gy WBI, the mRNA level of IL6 , TNF $\alpha$, IL- $1 \alpha$ and IL-1 $\beta$ were enhanced greatly compared to the control group, which is dependent on the radiation doses (Fig. 4). The expressions of IFN $\gamma$ were also measured but no significant difference was found (data not shown).

WBI resulted in $N F-\kappa B$ nuclear translocation in skin tissues

NF- $\kappa B$ induces expression of genes coding for many inflammatory cytokines, including IL-6, TNF $\alpha$, IL-1 $\beta$ etc [12]. And NF-KB p52/RelB also involves in the expression of chemokine, CCL19 and CCL21 $[12,13]$. We found that the nuclear NF-KB p65 (n-p65) was significantly higher than the control group at 0.5 and $1 \mathrm{~h}$ after 4 Gy irradiation, while the level of total 


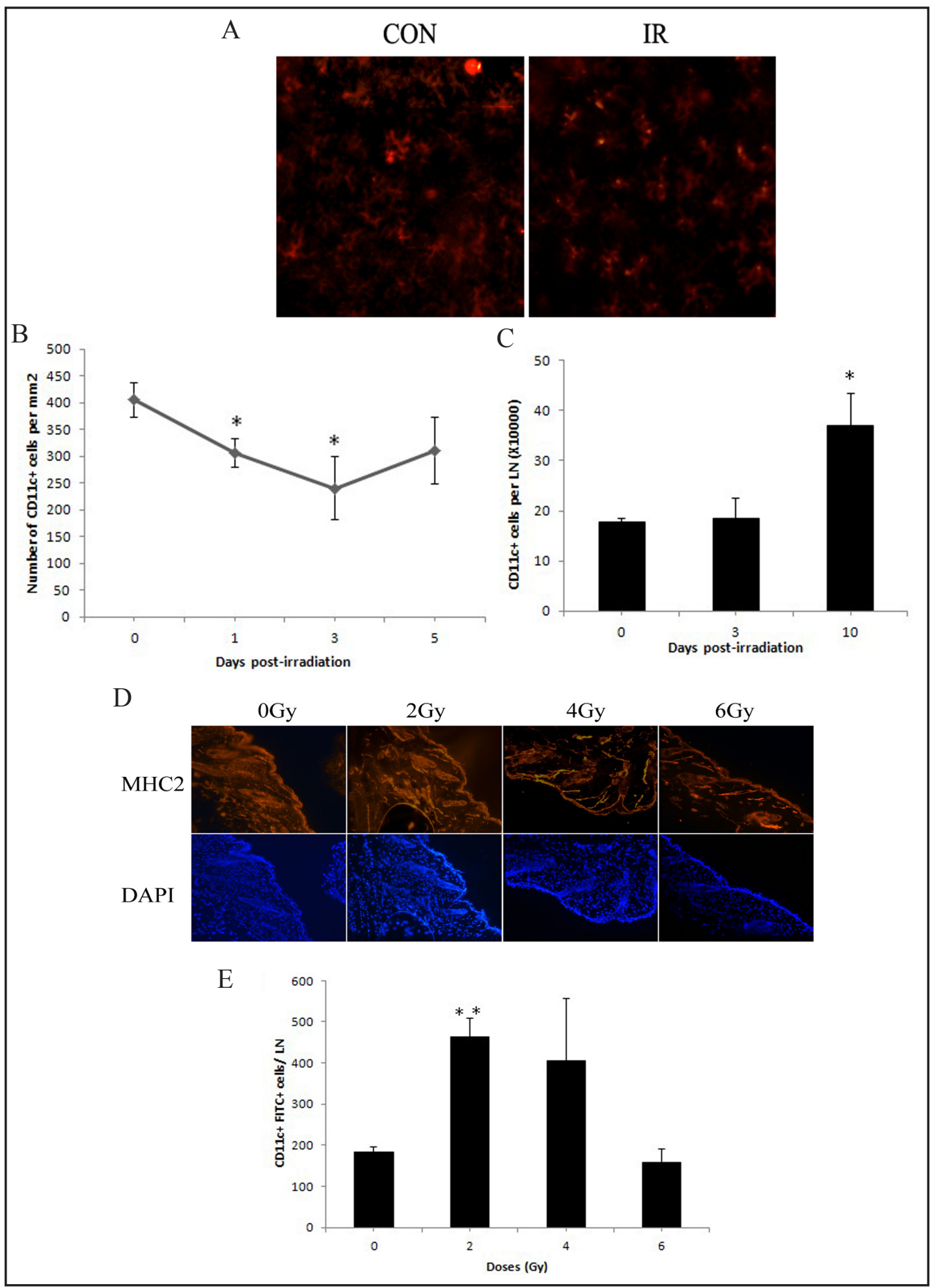

Fig. 1. WBI leaded to DC depletion. A: representative images of fluorescence of PE-CD11c staining epidermal sheets on D0 and D3 after 4Gy WBI. B: a bar graph of CD11 $\mathrm{c}^{+}$cell numbers per $\mathrm{mm}^{2}$ tissues. C: a bar graph of CD11 $\mathrm{c}^{+}$cells per LN. D: representative images of fluorescence of MHC $\alpha$ staining dermal sheets on D0 and D3 after 4Gy WBI. E: At 18h after FITC painting, inguinal LN cells were isolated and analyzed by using FCM, FITC ${ }^{+} \mathrm{CD} 11 \mathrm{c}^{+}$cells per LN were expressed in a bar graph. Values are given as mean \pm SEM $(n=5)$, ${ }^{* *} \mathrm{P}<0.01$ Vs the Control group. 
Fig. 2. WBI augmented DC migration from ear explants to CCL19. Cells migrated from the ear explant into the CCL19 containing medium was counted and expressed in a bar graph. Values are given as mean \pm SEM $(n=6),{ }^{*} P<0.05$ Vs the Control group.
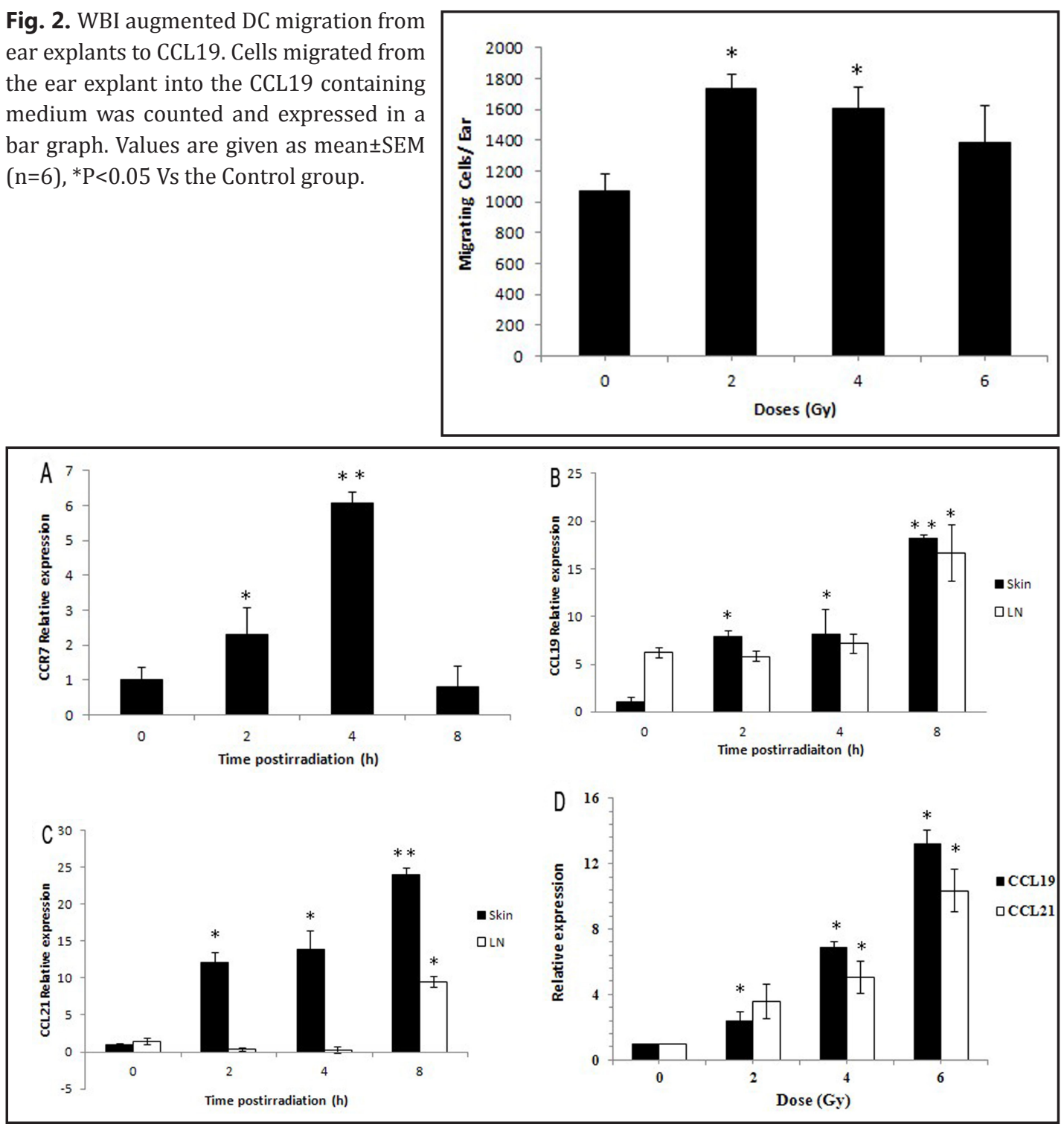

Fig. 3. WBI regulated the mRNA level of CCR7 and CCL19/21. Bar graphs of relative expressions of CCR7 (A), CCL19 (B, D) and CCL21 (C, D) in skin tissue at different time point after different doses of irradiation. Values are given as mean $\pm \operatorname{SEM}(n=5),{ }^{*} \mathrm{P}<0.05,{ }^{* *} \mathrm{P}<0.01$ Vs the Control group.

NF- $\kappa$ B p65 (t-p65) remained unchanged, indicating that WBI induced translocation of NF- $\kappa B$ p65 into the nuclear (Fig. 5). Therefore, the up-regulation of pro-inflammatory cytokines may be due to the activation of NF- $\kappa B$.

\section{$N F-\kappa B$ inhibition blocked the radiation induced depletion of $c D C$}

To determine whether NF- $\kappa B$ mediate depletion of $\mathrm{CDC}$ induced by irradiation, we used PDTC for the purpose of inhibition of NF- $\mathrm{\kappa B}$ in vivo according to previous reports [14]. Our data showed that the number of CD11c+ cells per $\mathrm{mm} 2$ in the epidermal reduced about $30 \%$ on 3rd day post-irradiation compared to the control group. While in the PDTC groups, the CD11c+ cells density increased in a dose dependent manner, and 100mg/kg PDTC almost totally rescued the DC reduction (Fig. 6A, B).

TLR4 is known to be related to the production of chemokine, CCL19/21, cytokines as well as the maturation and migration of DC [15]. In this study, we found that WBI caused 
Fig. 4. WBI caused changes of inflammatory cytokines. mRNA level of IL-6, TNF $\alpha$, IL- $1 \alpha$, and IL- $1 \beta$ was measured after different doses of WBI, the relative expressions were expressed in a bar graph. Values are given as mean \pm SEM $(\mathrm{n}=5),{ }^{*} \mathrm{P}<0.05,{ }^{* *} \mathrm{P}<0.01$ Vs the control group.

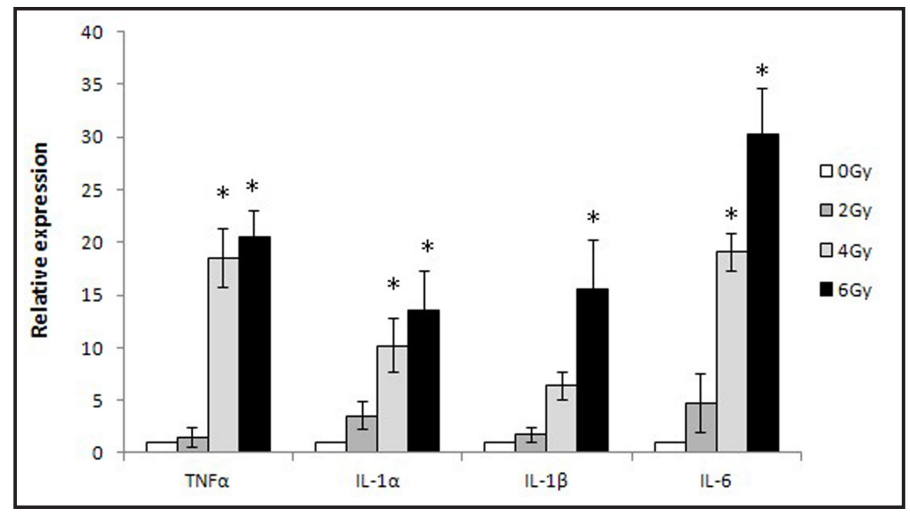

Fig. 5. WBI induced NF- $\kappa \mathrm{B}$ nuclear translocation. At $0,0.5,1,2 \mathrm{~h}$ after $4 \mathrm{~Gy}$ irradiation, total protein and nuclear protein was extracted from skin tissues. $\mathrm{NF}-\kappa \mathrm{B}$ p65 level in total protein and nuclear protein were analyzed by western blot. Actin level and lamin level were used to normalize loading for total protein and nuclear protein respectively.
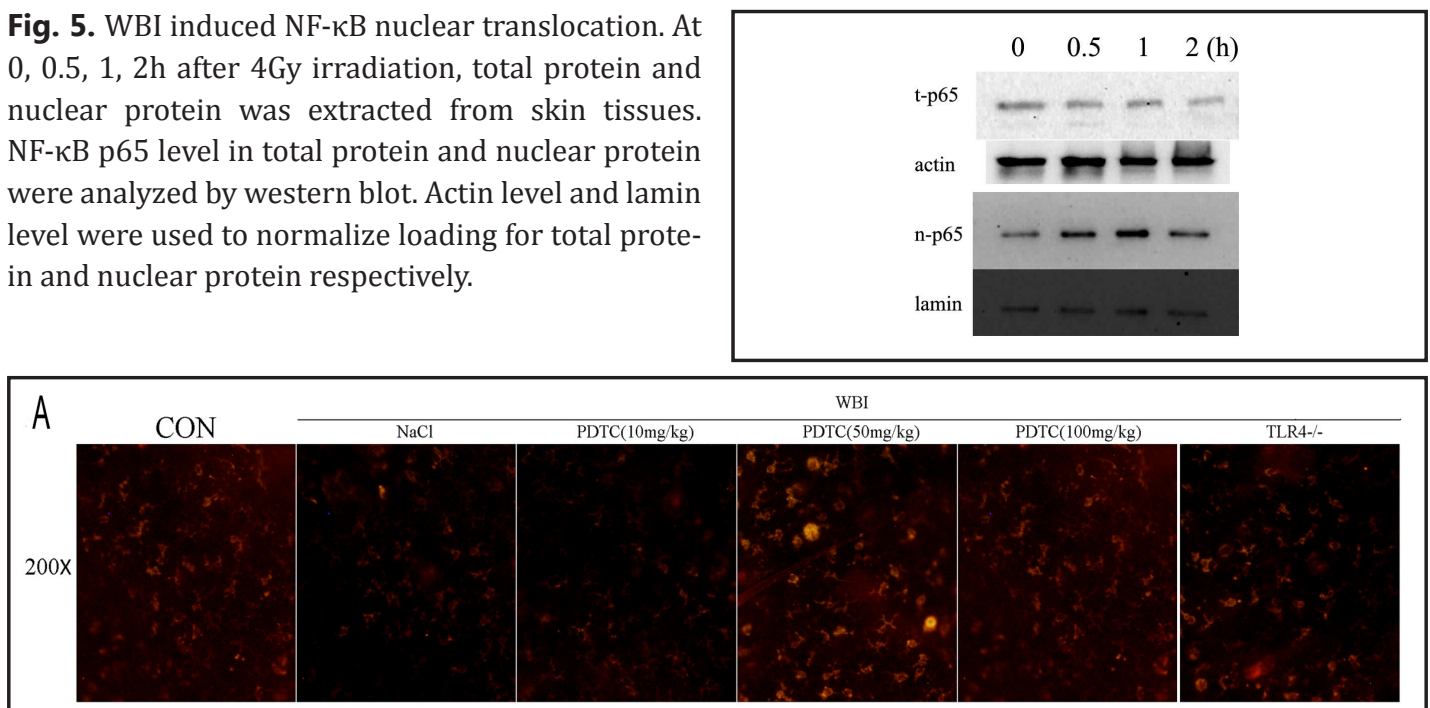

Fig. 6. NF- $\kappa \mathrm{B}$ inhibitor PDTC and TLR4-/- retained the reduction of DC density caused by irradiation. Mice were treated with $10 \mathrm{mg} / \mathrm{kg}, 50 \mathrm{mg} / \mathrm{kg}$ or $100 \mathrm{mg} / \mathrm{kg}$ PDTC at $1 \mathrm{~h}$ before 4Gy irradiation. TLR4-/- mice were also irradiated with the same dose of radiation. A: representative images of fluorescence of PE-CD11c staining epidermal sheets on D0 and D3 after WBI in different groups. B: a bar graph of $\mathrm{CD} 11 \mathrm{c}^{+}$cell numbers per $\mathrm{mm}^{2}$. Values are given as mean \pm SEM $(n=6),{ }^{*} \mathrm{P}<0.05$ Vs the single irradiated group.

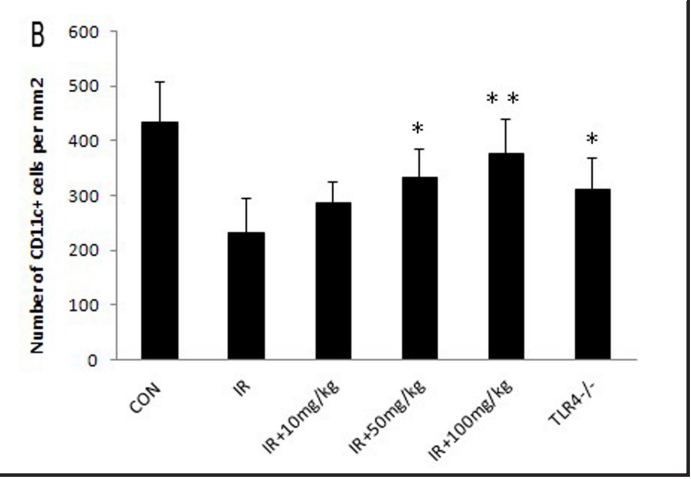

a reduction of the density of DC in TLR4-/- mice compared with the non-irradiated group (Fig. 6A, B), indicating that radiation induced depletion of DC was also related with TLR4 activation.

\section{Discussion}

Exposure to IR often leads to radiation dermatitis, which makes human body more susceptible to infections and damages [1]. Beside physical damages, the immunological damage of IR to the skin is important to human health and the recovery of radiation skin sickness. Cutaneous DC (CDC) are the most potent antigen presenting cells (APCs) that also act as orchestra of the immune response in the skin [16]. Understanding the influence of IR 
on $\mathrm{CDC}$ could provide sights into how the IR affects the skin immunity and the role of DC in radiation-induced immunosuppression. In this study, we found that different doses of WBI resulted in depletion of DC in the epidermal and dermal. WBI also augmented the migration capacity of cDC in FITC and chemokine induced models. WBI also lead to up-regulation of CCR7 and CCL19/21 in skin tissues. Multiple inflammatory cytokines were also greatly induced by WBI, and we also found WBI induced the translocation of NF- $\kappa$ B and radiationinduced decrease of DC was blocked by NF- $\mathrm{kB}$ inhibition and TLR4 knockout.

Our previous study had demonstrated that $\gamma$-irradiation inhibited DC migration to CCL19 in vitro [7]. It was reported that local irradiation triggered migration of LC in epidermal in a time and dose dependent manner [6]. Recently, Cummings et al [2] found that sub-lethal irradiation induced cutaneous DC migration to the draining LN. In this study, our data showed that WBI caused a depletion of cDC in epidermal and dermal. Since DC are quite resistant to ionizing radiation and it has also been demonstrated that DC could not be induced apoptosis even after 30Gy $\gamma$-irradiation [17]. Thus, we hypothesized that radiation triggered DC migration to the LN. Then we found that the number of DC in the draining LN increased after irradiation, which provided evidence for our hypothesis. The enhancement of IR on DC migration was also supported by a FITC-induced migration assay, and significant difference was observed at $18 \mathrm{~h}$ after irradiation. These data indicated that WBI induced cDC migration and the modulating effects of IR on DC is within $18 \mathrm{~h}$, or even much earlier. It was also demonstrated that more DC migrated from ear explants to the same concentration of CCL19 after WBI, which provided some clues that the concentration of chemokine in LN is not the only reason for the enhancement of migration capacity of DC. DC migration is a complicated process highly regulated by chemokine and their receptors, adhesion molecules, cytokines and other factors [18-20]. So next we measured the expressions of CCR7, its ligand CCL19/21 and some inflammatory cytokines.

CCR7 is an essential mediator for CDC migration to the draining LN under inflammatory and steady-state conditions [21]. Our data showed that the mRNA level of CCR7 in skin tissue was higher in the WBI group than the control group. As the ligands of CCR7, the level of CCL21 and CCL19 in skin and LN could greatly affect DC migration capacity and especially the migrating directions [22-25]. The mRNA level of CCL21 as well as CCL19 in the skin and the LN were greatly up-regulated after WBI. But the structure of skin tissue is so complicated that we could not conclude the concentration changes of chemokine along the whole routes of migration. These data suggests that the modulating effect on CCR7 and CCL21 may contribute to the enhancement of DC migration. As in previous study, we reported that IR down-regulated the CCR7 expression on DC in a dose dependent manner in vitro [7], inflammatory microenvironment induced by radiation might contribute a bigger part to the enhancement of DC migration than the direct effect on DC.

Acute radiation skin injury induces a formation of various cytokines and chemokine, such as IL-1 $\alpha$, IL-1 $\beta$, TNF $\alpha$, IL-6, IL-8, CCL10 and CCL2 $[1,26]$. And many of the DC maturation inducing factors such as IL-1 $\beta$, TNF $\alpha$, LPS, CpG and heat shock protein gp96 (Hsp gp96) have been well characterized $[4,12]$. And it was also measured in our study that the mRNA levels of IL- $1 \alpha$, IL- $1 \beta, T N F \alpha$ and IL- 6 in skin were all augmented after different doses of WBI. Almost all of these cytokines could induce DC maturation and migration: the situation seemed to be blurred. NF- $\kappa B$ induces expression of genes coding for many pro-inflammatory cytokines, including IL-6, TNF $\alpha$, IL-1 $\beta$ etc [27]. And IR can induce the activation of NF- $\kappa B$, which might be the reason for the up-regulation of these cytokines. It was reported that IL- 6 inhibited NF- $\kappa \mathrm{B}$ binding activity and CCR7 expression, while IL-1 $\beta$ and TNF $\alpha$ triggered DC migration $[13,28]$. NF- $\mathrm{\kappa B}$ p52/RelB also involves in the expression of chemokine, CCL19 and CCL21 [29]. Under normal conditions, most NF- $\kappa B$ subunits are sequestered in the cytoplasm by I- $\kappa$ B. Following activation, NF- $\kappa$ B subunits translocate into nucleus to regulate the expression of various genes [30]. In this study, we also detected NF- $\kappa B$ translocation to the nuclear after irradiation. PDTC was used to inhibit NF- $\kappa B$ activation in vivo in the previous studies [14, 31]. Our data showed that different concentrations of PDTC retained the DC density in the epidermal after irradiation, and $100 \mathrm{mg} / \mathrm{kg}$ PDTC almost abrogated the 
enhancement of migration by irradiation. This may provide some evidence that IR induce cDC migration depending on the NF- $\kappa \mathrm{B}$ activation. It has been reported that $\mathrm{DC}$ deprived from TLR4 deficient mice exhibited weaker migratory capacity [12]. TLR4 ligands induce DC maturation and migration and induce the expression of CCR7 through NF- $\mathrm{kB}$ pathway. In present study, we also found that IR did cause a reduction of DC in TLR4-/- mice, but the density is much higher than the irradiated groups of wild type mice, suggesting TLR4 as a role in mediating the radiation effects on DC migration. However, some other factors should also be determined in future study.

In conclusion, we reported different doses WBI caused DC depletion in the skin and augmented FITC and CCL19 induced DC migration, which may be attributed to induction of pro-inflammatory cytokines. And we also identified NF- $\kappa \mathrm{B}$ as a target of IR on DC migration in vivo. TLR4 activation was also related to radiation effects on DC migration. But the underlying mechanism is still to be clarified by future studies.

\section{Conflict of Interest}

The authors have no conflict of interest to disclose.

\section{Acknowledgements}

This work was supported by the grants from National Natural Science Foundation of China (No. 30970679 and No. 81001221).

\section{References}

1 Ryan JL: Ionizing radiation: the good, the bad, and the ugly. J Invest Dermatol 2012;132:985-993.

-2 Cummings RJ, Gerber SA, Judge JL, Ryan JL, Pentland AP, Lord EM: Exposure to Ionizing Radiation Induces the Migration of Cutaneous Dendritic Cells by a CCR7-Dependent Mechanism. J Immunol 2012;189:42474257.

- 3 Bennett CL, Van Rijn E, Jung S, Inaba K, Steinman R-M, Kapsenberg ML, Clausen BE: Inducible ablation of mouse Langerhans cells diminishes but fails to abrogate contact hypersensitivity. J Cell Biol 2005;169:569576.

4 Randolph GJ: Dendritic cell migration to lymph nodes: cytokines, chemokines, and lipid mediators. Semin Immunol 2001;13:267-274.

5 Cole S: Long-term effects of local ionizing radiation treatment on Langerhans cells in mouse footpad epidermis. J Invest Dermatol 1986;87:608-612.

6 Cummings RJ, Mitra S, Foster TH, Lord EM: Migration of skin dendritic cells in response to ionizing radiation exposure. Radiat Res 2009;171:687-697.

7 Liu C, Lin J, Zhao L, Yang Y, Gao F, Li B, Cui J, Cai J: Gamma-ray irradiation impairs dendritic cell migration to CCL19 by down-regulation of CCR7 and induction of cell apoptosis. Int J Biol Sci 2011;7:168-179.

$>8$ Robbiani DF, Finch RA, Jager D, Muller WA, Sartorelli AC, Randolph GJ: The leukotriene C(4) transporter MRP1 regulates CCL19 (MIP-3beta, ELC)-dependent mobilization of dendritic cells to lymph nodes. Cell 2000;103:757-768.

-9 Ratzinger G, Stoitzner P, Ebner S, Lutz MB, Layton GT, Rainer C, Senior RM, Shipley JM, Fritsch P, Schuler G, Romani N: Matrix metalloproteinases 9 and 2 are necessary for the migration of Langerhans cells and dermal dendritic cells from human and murine skin. J Immunol 2002;168:4361-4371.

10 Haridas S, Bowers M, Tusano J, Mehojah J, Kirkpatrick M, Eburnham DK: The impact of Meth A fibrosarcoma derived EMAP II on dendritic cell migration. Cytokine 2008;44:304-309. 
11 Lin J, Liu C, Gao F, Mitchel R-E, Zhao L, Yang Y, Lei J, Cai J: MiR-200c enhances radiosensitivity of human breast cancer cells. J Cell Biochem 2013;114:606-615.

12 Hatfield P, Merrick A, Harrington K, Vile R, Bateman A, Selby P, Melcher A: Radiation-induced cell death and dendritic cells: potential for cancer immunotherapy? Clin Oncol (R Coll Radiol) 2005;17:1-11.

13 Hegde S, Pahne J, Smola-Hess S: Novel immunosuppressive properties of interleukin-6 in dendritic cells: inhibition of NF-kappaB binding activity and CCR7 expression. FASEB J 2004;18:1439-441.

14 Sasaki H, Zhu L, Fukuda S, Maulik N: Inhibition of NF kappa B activation by pyrrolidine dithiocarbamate prevents in vivo hypoxia/reoxygenation-mediated myocardial angiogenesis. Int J Tissue React 2000;22:93100.

15 Alaniz L, Rizzo M, Piccioni F, Aquino JB, Malvivini M, Atorrasagasti C, Bayo J, Echeverria I, Sarobe P, Mazzolini G: Low molecular weight hyaluronan preconditioning of tumor-pulsed dendritic cells increases their migratory ability and induces immunity against murine colorectal carcinoma. Cancer Immunol Immunother 2011;60:1383-1395.

16 Banchereau J, Steinman RM: Dendritic cells and the control of immunity. Nature 1998;392:245-252.

17 Rao V, Saunes M, Jorstad S, Moen T: In vitro experiments demonstrate that monocytes and dendritic cells are rendered apoptotic by extracorporeal photochemotherapy, but exhibit unaffected surviving and maturing capacity after 30 Gy gamma irradiation. Scand J Immunol 2008;68:645-651.

18 Martin-Fontecha A, Lanzavecchia A, Sallusto F: Dendritic cell migration to peripheral lymph nodes. Handb Exp Pharmacol 2009;188:31-49.

19 Colvin B-L, Matta BM, Thomson AW: Dendritic cells and chemokine-directed migration in transplantation: where are we headed? Clin Lab Med 2008;28:375-384.

20 Alvarez D, Vollmann EH, von Andrian UH: Mechanisms and consequences of dendritic cell migration. Immunity 2008;29:325-42.

-21 Ohl L, Mohaupt M, Czeloth N, Hintzen G, Kiafard Z, Zwirner J, Blankenstein T, Henning G, Forster R: CCR7 governs skin dendritic cell migration under inflammatory and steady-state conditions. Immunity 2004;21:279-288.

22 Johnson LA, Jackson DG: Inflammation-induced secretion of CCL21 in lymphatic endothelium is a key regulator of integrin-mediated dendritic cell transmigration. Int Immunol 2010;22:839-849.

23 Murphy, PM: Double duty for CCL21 in dendritic cell trafficking. Immunity 2010;32:590-592.

24 Hansson M, Lundgren A, Elgbratt K, Quiding-Jarbrink M, Svennerholm A-M, Johansson E-L: Dendritic cells express CCR7 and migrate in response to CCL19 (MIP-3beta) after exposure to Helicobacter pylori. Microbes Infect 2006;8:841-850.

25 Jang M-H, Sougawa N, Tanaka T, Hirata T, Hiroi T, Tohya K, Guo Z, Umemoto E, Ebisuno Y, Yang B-G, Seoh JY, Lipp M, Kiyono H, Miyasaka M: CCR7 is critically important for migration of dendritic cells in intestinal lamina propria to mesenteric lymph nodes. J Immunol 2006;176:803-810.

26 Luckey TD: Radiation hormesis: the good, the bad, and the ugly. Dose Response 2006;4:169-190.

27 Pahl HL: Activators and target genes of Rel/NF-kappaB transcription factors. Oncogene 1999;18:68536866.

28 Stoitzner P, Zanella M, Ortner U, Lukas M, Tagwerker A, Janke K, Lutz MB, Schuler G, Echtenacher B, Ryffel B, Koch F, Romani N: Migration of langerhans cells and dermal dendritic cells in skin organ cultures: augmentation by TNF-alpha and IL-1beta. J Leukoc Biol 1999;66:462-470.

29 Muller JR, Siebenlist U: Lymphotoxin beta receptor induces sequential activation of distinct NF-kappa B factors via separate signaling pathways. J Biol Chem 2003;278:2006-2012.

-30 Chen T, Guo J, Yang M, Han C, Zhang M, Chen W, Liu Q, Wang J, Cao X: Cyclosporin A impairs dendritic cell migration by regulating chemokine receptor expression and inhibiting cyclooxygenase-2 expression. Blood 2004;103:413-421.

-31 Liu SF, Ye X, Malik AB: Inhibition of NF-kappaB activation by pyrrolidine dithiocarbamate prevents In vivo expression of proinflammatory genes. Circulation 1999;100:1330-1337. 